\title{
Jhering, Kohler und das Erbe der "Pandektenlehrbücher"
}

\author{
"Er muß es sich schon gefallen lassen, wenn ich ihm dahin nicht folge, es steckt noch zu viel von der \\ alten Jurisprudenz aus den 'Pandektenlehrbüchern' in mir, um die neue Aera der Jurisprudenz, die er \\ uns erschließt, mitmachen zu können"1.
}

Josef Kohler konnte diese klare Zurückweisung, die der hochberühmte Jhering dem so selbstbewussten jungen Würzburger Professor 1883 zukommen ließ, nie vergessen. Über Jahre hinweg überzog er Jhering mit Hass ${ }^{2}$ und noch 1919, kurz vor seinem Tod, attestierte Kohler dem bereits 1892 Gestorbenen, "daß er für die ganze Technik des Dramas kein Verständnis hatte"3.

Ausgangspunkt war die von Jhering im "Kampf ums Recht" 1872 vertretene Ansicht, dass Shylock in Shakespeares "Kaufmann von Venedig" Unrecht geschehen sei, als man seinem vom Gericht als rechtmäßig anerkannten Schuldschein die Vollstreckung unmöglich machte. Seitens des Richters sei es "ein elender Winkelzug, ein kläglicher Rabulistengriff" gewesen, "dem Manne, dem er bereits das Recht zugesprochen hatte, vom lebenden Körper ein Pfund Fleisch auszuschneiden, das damit verbundene Vergießen des Blutes zu versagen"4. Jhering störte die richterliche Begründung, die sich nicht juristischer, sondern außerrechtlicher Argumente bediente. Man habe den Schuldschein für sittenwidrig erklären müssen. Tat man das nicht, musste die Vollstreckung gestattet werden. Kohler hielt dem das Rechtsgefühl des Richters entgegen, das unaufhebbar seine Entscheidung determiniere. Der von Jhering übersehen Faktor sei "das Rechtsbewusstsein des Richters, der im Richter lebende Rechtsinstinkt, der sich noch nicht zur vollständig klaren Erkenntnis herausgearbeitet hat und sich daher hinter den Scheingründen des weisen Daniel verbirgt"5.

Der Streit zwischen Jhering und Kohler um Shylock faszinierte bereits Zeitgenossen ${ }^{6}$ und

1 Rudolph v. Jhering, Vorwort zur 7. Aufl. des "Der Kampf ums Recht", Wien 1884, datierend vom 3. Oktober 1883.

2 Vgl. nur Josef Kohler, Nachwort zu Shakespeare vor dem Forum der Jurisprudenz, Würzburg 1884, passim; ders., Die Wiedergeburt der Rechtsphilosophie, in: AfRWPh 2, 1908/09, S. 446; ders., Recht und Persönlichkeit in der Kultur der Gegenwart, Stuttgart 1914, S. 23.

3 Josef Kohler, Shakespeare vor dem Forum der Jurisprudenz, 2. Aufl. Berlin 1919, S. 6.

4 Rudolph Jhering, Der Kampf ums Recht, hier nach vierter Aufl. Wien 1874, S. 59 Anm. *)

5 Josef Kohler, Shakespeare vor dem Forum der Jurisprudenz, Würzburg 1883, S. 83.

6 Vgl. noch nur zu Jhering: August Pietscher, Jurist und Dichter. Versuch einer Studie über Jhering's "Kampf um's Recht" und Shakespeare's "Kaufmann von Venedig", Dessau 1881; dann Ludwig Gumplowicz, Rez. Jos. Kohler. Shakespeare vor dem Forum der Jurisprudenz. In: Grünhuts Zeitschrift 1885. S. 242 - 244; A. S., Shakespeare vor dem Forum der Jurisprudenz Jos. Kohler. In: KritV Neue Folge, Vol. 7 (26), Supplement 1884. S. 744 - 748; Richard Schroeder, J. Kohler, Shakespeare vor dem Forum der Jurisprudenz. Würzburg 1883. In: ZRG 6.1 (1885), S. 220 f.; Stutz, Ulrich: Josef Kohler, 


\section{H.-P.HAFERKAMP}

löste besonders in den USA ${ }^{7}$ Diskussionen um Shakespeare und das Recht aus ${ }^{8}$. Juristen interessierte die Debatte vor allem, weil man beiden Protagonisten visionäre Kraft attestierte $^{9}$. In der Argumentation Kohlers wurde immer wieder die Entdeckung volitiver Momente in der richterlichen Tätigkeit als zukunftsweisende Entlarvung naiver Bindungsphantasien gefeiert ${ }^{10}$. Es war Kohler selbst, der 1919 im Vorwort zur zweiten Auflage seines Shakespeare-Buches seine Äußerungen des Jahres 1883 als "Morgenröthe des Freirechtsbewegung"11 empfand - eine Selbststilisierung, die Freirechtler wie Hermann Kantorowicz freilich zurückwiesen ${ }^{12}$, wohl weil ihnen Kohlers Verweis auf "Volksinstincte"13 verdächtig nach der alten Volksgeistmystik ${ }^{14}$ klang, die man philosophisch und soziologisch als Dilettantismus empfand ${ }^{15}$. Kantorowicz benannte dagegen in Jhering eine prägende Gestalt der Freirechtsbewegung ${ }^{16}$ und sah wie viele Andere im "Kampf ums Recht" den Ursprung einer soziologisch denkenden Jurisprudenz ${ }^{17}$.

Weniger im Blick dieser Diskussionen waren die rechtspolitischen Konzepte, die in diesem Streit durchschienen. Diesem Ziel dienen die nachfolgenden Anmerkungen. Rechtspolitisch

Shakespeare vor dem Forum der Jurisprudenz, 2. Auflage mit einem Bildnis des Verfassers. Berlin und Leipzig, Walther Rothschild, 1919. S. 389 ff.

7 Überblick bei Owen Hood Philipps, Shakespeare and the Lawyers, London/ New York 1972. S. $92 \mathrm{ff}$.

8 Etwa die Diskussion um die Frage, ob Shakespeares Ansicht dem venezianischen Recht entsprach zwischen Kohler und Th. Niemeyer: The Judgement against Shylock in the merchant of venice. In: Michigan Law Review Vol. 14 Nr. 1 (Nov. 1915), S. 20 - 36; dagegen Julius Hirschfeld: "Portia's Judgment and German Jurisprudence," Law Quarterly Review 118 (1914); daneben geht es um das Judentum von Shylock, vgl. etwa Anton Schütz, Structural Terror: A Shakespearean Investigation, in: Peter Goodrich, Lior Barshack, Anton Schütz (Hgg.). Law, Text, Terror. Abingdon 2006. S. 71 ff. bes. 82 91. Dabei kommt es zu interessanten Gemeinschaftsprojekten zwischen Rechtshistorikern und Literaturwissenschaftlern vgl. etwa die Beiträge von Klaus Grubmüller, Uwe Diederichsen und Theodor Wolpers, in: Ulrich Mölk (Hg.). Literatur und Recht: Literarische Rechtsfälle von der Antike bis zur Gegenwart. Göttingen: Wallstein, 1996. 186-228.

9 Vgl. etwa Peter Klein, Rez. Shakespeare vor dem Forum der Jurisprudenz, 2. Aufl., in: ARWPh 13, 1919/20, S. 225.

10 Vgl. nur Günter Spendel, Josef Kohler. Bild eines Universaljuristen, Heidelberg 1983, S. 32.

11 Kohler, Shakespeare (Fn. 3), 1919, S. VI.

12 Kantorowicz, Rez. Kohler, Shakespeare vor dem Forum der Jurisprudenz, 2. Aufl. 1919, in: HZ 128, 1923, S. 454.

13 Kohler, Shakespeare (Fn. 3), 1883, S. 87.

14 Vgl. Rudolf Stammler, Über die Methode der geschichtlichen Rechtstheorie, in ders. u. Theodor Kipp (Hgg.), Festgabe zu Bernhard Windscheids fünfzigjährigem Doktorjubiläum, Halle 1888, S. 6.

15 Vgl. nur Anna Babette Stier, "Richtiges Recht" zwischen Entwicklungs- und Kulturgedanken. Prinzipien der Rechtsgestaltung in der Rechtstheorie um 1900, Berlin 2006, S. 122 f., 158 f.; Hasso Hofmann, A treatise of Legal Philosophy and General Jurisprudence, Vol. 9: A History oft he Philosophy of Law in the Civil Law World, 1600-1900, Dordrecht u. a. 2009, S. 338; schon die Historische Schule hatte sich diesen Vorwurf ausgesetzt gesehen, vgl. Hans-Peter Haferkamp, Die Historische Rechtsschule, Frankfurt a. M. 2018, S. $156 \mathrm{ff}$.

16 Vgl. Hermann Kantorowicz, in: Jherings Bekehrung, in: DRiZ 1914, Sp. 87.

17 So vor allem Roscoe Pound, vgl. Nils Jansen/Mathias Reimann, Begriff und Zweck in der Jurisprudenz - Ein Geburtstagsblatt für Rudolf von Jhering, in: ZEuP 2018, S. 122 f.; in Deutschland vor allem Helmut Schelsky, Das Jhering-Modell des sozialen Wandels durch Recht, Jahrbuch für Rechtssoziologie und Rechtstheorie 3, 1972, S. 47 ff. 


\section{JHERING, KOHLER UND DAS ERBE DER "PANDEKTENLEHRBÜCHER"}

ging es in diesem Streit um die Aufgabe, die man dem Richter im Privatrecht zumaß. Jherings Hinweis auf das Erbe der "Pandektenlehrbücher" und seine beißende Ironie gegenüber Kohlers Gerede von einer im Rechtsgefühl wirklich werdenden "welthistorischen Nothwendigkeit"18 waren Ausdruck einer dezidiert privatrechtsliberalen Position, die Kohler so nicht teilte.

Kohlers Argumentation war stark richterrechtlich orientiert - nicht untypisch für einen ehemaligen Richter. Er machte die Justiz zum Vollstrecker des zeitgenössischen Gerechtigkeitsbewusstseins, wenn die Gesetzgebung nicht reagierte: "Wo es aber die Gesetzgebung nicht thut, da thut es die Rechtspflege, sie thut es, oft völlig unbewusst und ahnungslos, aber sie thut es. Keine Rechtspflege der Welt, und wäre sie die gelehrteste und abstracteste, kann sich dem Rechtsbewusstsein des Volkes entziehen"19. Kohler setzte auf richterliche Intuition als entscheidungstragend - auch und gerade gegen das Gesetz. Nach Jherings Kritik wurde er deutlicher. In welthistorischer Perspektive berief er sich 1883 zunächst auf einen schlichten Erfahrungssatz, eine "Erscheinung, die das Rechtsleben aller Völker bietet"20. 1886 brach er das Thema in seinem bekannten Aufsatz über die "Interpretation von Gesetzen" auf den nach $\S 1$ GVG gebundenen Richter des Kaiserreichs herunter. Auch hier habe der Richter in Fällen, "in welchen die gesetzlichen Bestimmungen in ihrer richtigen Interpretation dem Rechtsbewußtsein nicht mehr entsprechen und das Gesetzesrecht in Reibung tritt mit den Culturinteressen" den Gesetzeswortlaut "so restrictiv zu erklären, dass sich die ganze Bestimmung zuletzt in ein Nichts auflöst und juristisch eliminiert wird". Dass "hier nicht mehr Auslegung im wahren Sinne vorliegt, dass hier unter der Form der Auslegung sich in der That eine Rechtsneubildung, ein Rechtsfortschritt verbirgt, dass auf diese Weise der gewohnheitsrechtliche Gang der Rechtsfortbildung seinen langsamen stetigen Fortgang nimmt", war ihm weiterhin eine Wahrheit, die er auf seine Erörterungen zu Shylock stützte $^{21}$. Der Gesetzeswortlaut wurde damit zum bloßen Blankett der richterlichen Gewohnheitsrechtsbildung. Dies funktionierte rechtsquellentheoretisch, weil er noch ganz auf dem Boden der Gewohnheitsrechtslehre Puchtas ${ }^{22}$ stand. Für Kohler blieb es Puchtas "unsterbliches Verdienst", das "geistige Element in der Gewohnheit betont zu haben"23. Im Gewohnheitsrecht verwirklichten sich für Kohler "die soziale Rechtsanschauung von selbst"24. Völlig unrichtig sei daher die Meinung "als ob das Gewohnheitsrecht einfach darin begründet sei, daß etwas oft geschieht, sodaß daraus zu schließen sei, daß es immer geschehen

\footnotetext{
18 Jhering, Kampf (Fn. 1), Vorwort zur 7. Aufl. 1883.

19 Josef Kohler, Shakespeare (Fn. 13), 1883, S. 87.

20 Josef Kohler, Rechtsgeschichte und Weltentwicklung, in: Zeitschrift für vergleichende Rechtswissenschaft 5, 1884, S. 327,

21 Josef Kohler, Über die Interpretation von Gesetzen, in: (Grünhuts) Zeitschrift für das Privat- ind Öffentliche Recht der Gegenwart 13, 1886, S. 59.

22 Vgl. hierzu Hans-Peter Haferkamp, Georg Friedrich Puchta und die ,Begriffsjurisprudenz', Frankfurt a. M., 2004, S. $173 \mathrm{ff}$.

23 Kohler, Lehrbuch des Bürgerlichen Rechts, Bd. 1, Berlin 1906, S. 106 Fn. 2).

24 Kohler, Lehrbuch (Fn. 23), 1906, S. 106.
} 


\section{H.-P.HAFERKAMP}

müsse"25. Indem der Richter Gesetze teilweise abwegig auslegte, kam in ihm "eine spontane soziale Bildung" zum Ausdruck. Ein Gerichtsgebrauch liege daher dann vor, "wenn das Gericht eine bestimmte Auslegung deswegen gibt, weil sie seinem instinktiven Rechtssinn entspricht. Der Auslegung muß ein aus dem Rechtsgefühl des Richters hervorgehendes Gestaltungsstreben zugrunde liegen, ein unbewußtes Bestreben, das nur der dritte Beobachter erkennt. ... Die Auslegung kann in einem solchen Falle sehr unrichtig sein, ganz gewagt, und den Auslegungsregeln widersprechend: sie wird eben deswegen zum Rechte werden, weil sie der Ausdruck der abweichenden Rechtsüberzeugung ist"26.

1906 fügte Kohler diesen Äußerungen hinzu: "Vor 20 Jahren allerdings gab es in Deutschland Männer, die ein gewisses Ansehen genossen und dieses erste Prinzip der gewohnheitsrechtlichen Rechtsbildung verkannten"27. Noch 16 Jahre nach Jherings Tod betrieb Kohler seine Shylock-Fehde mit Jhering. Zwar hatte man in der Shylockkontroverse nicht direkt über das Gewohnheitsrecht diskutiert, aber Kohlers Einschätzung traf Jherings Ansicht durchaus. Seine Rechtsentstehungslehre im "Kampf ums Recht" hatte untergründig genau Puchtas Gewohnheitsrechtslehre angegriffen. Dessen Vorstellung, dass das Recht "unvermerkt und schmerzlos" durch die "still wirkende Kraft der Wahrheit" allmählich die "Überzeugung" der "Gemüther" erschließe, vertröste den Menschen auf einem "Gebiete, wo er handeln soll, und mit vollem, klarem Bewusstsein des Zweckes und mit Aufbietung aller seiner Kräfte handeln soll, darauf, dass die Dinge sich von selbst machen, dass er am besten thue, die Hände in den Schoss zu legen und vertrauensvoll abzuwarten, was aus dem Urquelle des Rechts: der nationalen Rechtsüberzeugung nach und nach an's Tageslicht

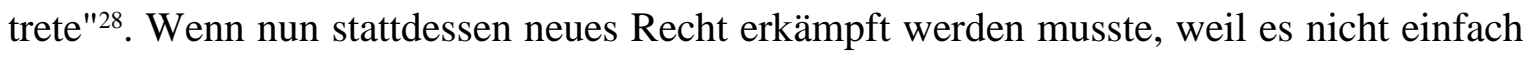
von selbst kam, dann war für Jherings Position charakteristisch, dass der Richter nicht befugt war, diesen Kampf zu führen. Puchtas Gewohnheitsrechtslehre war für ihn bloße Tarnung für ungesteuertes Richterrecht. Früh bereits hatte sich Jhering von den Freiheiten, die die Volksgeistlehre dem Richter gewährte ${ }^{29}$, verabschiedet. In seinen Vorlesungen hatte Jhering bereits 1858 Distanz zu Puchta in dieser Frage aufgebaut. Puchta gehe in seiner Betonung der richterlichen Überzeugung als Träger des Gewohnheitsrechts "entschieden zu weit". Gewohnheitsrecht beruhte für Jhering nicht auf Überzeugungen, sondern auf der Übung: "Macht der Thatsache auf dem Gebiete des R(echt)s ist eben der Grund"30. Wenn Jhering sich 1883 also auf das Erbe der Pandektenlehrbücher berief, dann nicht in dieser Frage $^{31}$. Eine Entscheidung musste sich bewähren, um sich auf Gewohnheitsrecht berufen

\footnotetext{
Kohler, Lehrbuch (Fn. 23), 1906, S. 106.

Kohler, Lehrbuch (Fn. 23), 1906, S. 111.

Kohler, Lehrbuch (Fn. 23), 1906, S. 113 Fn. 1).

Jhering, Kampf (Fn. 4), 1874, S. 11.

Vgl. Haferkamp, Historische Rechtsschule (Fn. 15), 2018, S. 284 ff.

Rudolf v. Jhering, Pandektenvorlesung nach Puchta, hg. und kommentiert von Christian Jäde, Göttingen 2008, S. 63.

31 Puchtas Gewohnheitsrechtslehre war freilich zu keinem Zeitpunkt ohne Kritiker, vgl. nur Jan Schröder, Zur Theorie des Gewohnheitsrechts zwischen 1850 und 1930, in: ders., Rechtswissenschaft in der Neuzeit, Tübingen 2010, S. 547 ff.
} 


\section{JHERING, KOHLER UND DAS ERBE DER "PANDEKTENLEHRBÜCHER"}

zu können. Damit waren, genau genommen, alle früheren Entscheidungen, wenn sie - wie bei Kohler postuliert - gegen den Gesetzeswortlaut getroffen wurden, rechtswidrig.

Jhering band den Richter erstaunlich streng an das Gesetz ${ }^{32}$. Das bedeutete keineswegs Naivität gegenüber den richterlichen Freiräumen in der Entscheidungsfindung, die Kohler so stark machte. Jhering ironisierte die Vorstellung, dass das richterliche Denken durch das Gesetz überflüssig gemacht werden soll", vergleichbar einem Verdauungsprozess: "vorn wird der Fall in die Urteilsmaschine hineingeschoben, hinten kommt er als Urteil wieder heraus"33. Gerade weil "der Kopf des Richters ... sich durch den Gesetzgeber nicht ersetzen" lasse ${ }^{34}$, konzipierte Jhering Gesetzesbindung als eine richterliche Tugend: "Gehorsam gegen

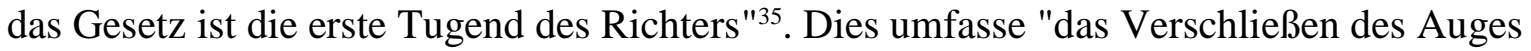
gegen jedes Ansehen der Person ... das Verschließen des Ohrs gegen die Klagen der Armen und Elenden und den Jammer der Angehörigen, denen der Richterspruch den Gatten und Vater rauben soll. Nicht den schlechten Menschen gilt es zu unterdrücken, sondern den guten, und das ist die schwerste Prüfung, welche der Dienst der Gerechtigkeit mit sich bringt" und zwar selbst dann, wenn "das Gesetz, welches der Richter vollziehen soll, mit dessen eignem Rechtsgefühl in schneidendem Widerspruch steht"36. Der Richter solle nichts sein, als "das lebendig gewordene, in seiner Person der Sprache teilhaftig gewordene Gesetz" ${ }^{37}$. Daher sein Eintreten für Shylock.

Man hat diese Position Jherings immer wieder in die große Schublade ${ }^{38}$ des "Positivismus"39 geschoben und damit etwas als erkenntnistheoretische Position gebrandmarkt, was zunächst ein ganz bewusstes politisches Konzept war ${ }^{40}$. Dies wird bereits deutlich mit Blick auf das Strafrecht. Hier zeigte sich Jhering nämlich erstaunlich offen gegenüber richterlicher Rechtssetzung. Keine Spur von Gesetzespositivismus. Von nulla poena sine lege wollte er hier nichts wissen. Gegenüber Verbrechern, deren Taten nicht gesetzlich verboten seien, machte er die Rechtsprechung zum Vollstrecker des Volksbewusstseins und stellte einen

32 Hierzu die glänzende Analyse von Ulrich Falk, Von Dienern des Staates und von anderen Richtern. Zum Selbstverständnis der deutschen Richterschaft im 19. Jahrhundert, in: André Gouron et. al., Europäische und amerikanische Richterbilder, Frankfurt a. M. 1996. S. $271 \mathrm{ff.}$

33 Rudolph v. Jhering, Der Zweck im Recht, Bd. 1, Leipzig 1877, S. 394.

34 Jhering, Zweck I (Fn. 34), 1877, S. 395.

35 Jhering, Zweck I (Fn. 34), 1877, S. 409.

36 Jhering, Zweck I (Fn. 34), 1877, S. 412.

37 Jhering, Zweck I (Fn. 34), 1877, S. 387.

38 Zu diesem Problem Hans-Peter Haferkamp, Positivismen als Ordnungsbegriffe einer Privatrechtsgeschichte des 19. Jahrhunderts, in: Okko Behrends u. Eva Schumann IHgg.), Franz Wieacker - Historiker des modernen Privatrechts, Göttingen 2010, S. 181 ff.

39 Vgl. etwa Richard H. Weisberg, Lawyers and Judges address Shylocks case, in: E. Nahshon, M. Shapiro (Hgg.). Wrestling with Shylock. Jewish Responses to the Merchant of Venice. 2007. Pp. 105 - 116; Okko Behrends, Das "Rechtsgefühl" in der historisch-kritischen Rechtstheorie des späten Jhering, in: Jhering, Über die Entstehung des Rechtsgefühls, 1986, S. 81 f. u. ö.; zutreffende Kritik bei Rückert, Der Geist des Rerchts in Jherings "Geist" und Jherings "Zweck", in: Rg 5, 2004, S. 139.

Vgl. Falk, Von Dienern des Staates, 1996, S. 273 ff. 


\section{H.-P.HAFERKAMP}

"höchsten Gerichtshof über das Gesetz": "Das unbefangene Rechtsgefühl des Volkes verlangt auch hier Bestrafung, und ich meinerseits schließe mich dem vollkommen an"41. Für das Strafrecht galt als höchstes Ziel "nicht die Fernhaltung der Willkür, sondern die Verwirklichung der Gerechtigkeit", wohingegen "in der Zivilrechtspflege verlangen wir die unverbrüchliche Anwendung des Gesetzes und nehmen die etwaigen Härten und Unbilligkeiten in Kauf"42. Jherings Beharren darauf, dass Shylock Unrecht geschehen war, weil der Richter einen für legal erklärten Schuldschein nicht anerkannt habe, war also eine dezidiert privatrechtliche Perspektive.

Kohler sah das Privatrecht bei dem Richter dagegen in guten Händen, weil er sowieso die Trennung zwischen Staat und Gesellschaft nicht als entscheidend empfand. Im Privatrecht war dem Einzelnen ein Raum für Rechtsgestaltung gewährt, damit "er reichlich mitarbeiten darf und mitarbeiten soll an den Fortschritten des Rechts" ${ }^{43}$. Privatrecht war nicht ursprünglich, sondern abgeleitet, zu messen an seinem kulturellen Wert ${ }^{44}$. Die Einschätzung dieses Wertes habe, so meinte er 1906, "im Laufe der Zeit sehr gewechselt" und heute gelte, dass "das soziale Gebiet tief in das menschliche Leben eindringt und ihm nur sehr teilweise die Herrschaft überlässt"45. Auch Privatrecht war dem Fortschritt der Gesellschaft unterworfen. Subjektive Rechte konnten diesen Fortschritt nicht aufhalten. "Kein Fortschritt in der Welt, und wäre es der Fortschritt des Rechts, ist möglich ohne individuelle Ungerechtigkeit; jeder grosse Fortschritt der Gesellschaft schreitet über Leichen hinweg"46. Ein Richter, dessen Rechtsgefühl die "unbewusste Vernünftigkeit in der Kulturgeschichte"47 umsetzte, war Konsequenz aus Kohlers Vorstellung einer letztlich nur ex post verstehbaren "göttlichen Vernunft"48 in der Geschichte der Völker. "Dieser Prozeß vollzieht sich nicht durch einen äusseren Machtspruch: sondern durch die Gewalt der die Menschengeschichte lenkenden geistigen Kräfte"49. Der Richter war Medium des Weltgeistes, dem subjektive Rechte nicht in den Weg treten konnten.

Wenn auch Jhering sah, dass subjektive Rechte dem Wandel oft zum Opfer fielen (das "Gewordene aber muss dem neuen Werden weichen"50), weshalb "überall aber zertretene Rechte den Weg, den das Recht dabei gewandelt hat"51 bezeichnen würden, dann fand er darin nicht die Tätigkeit des Weltgeistes, sondern den Angriff des Staates auf die Rechte

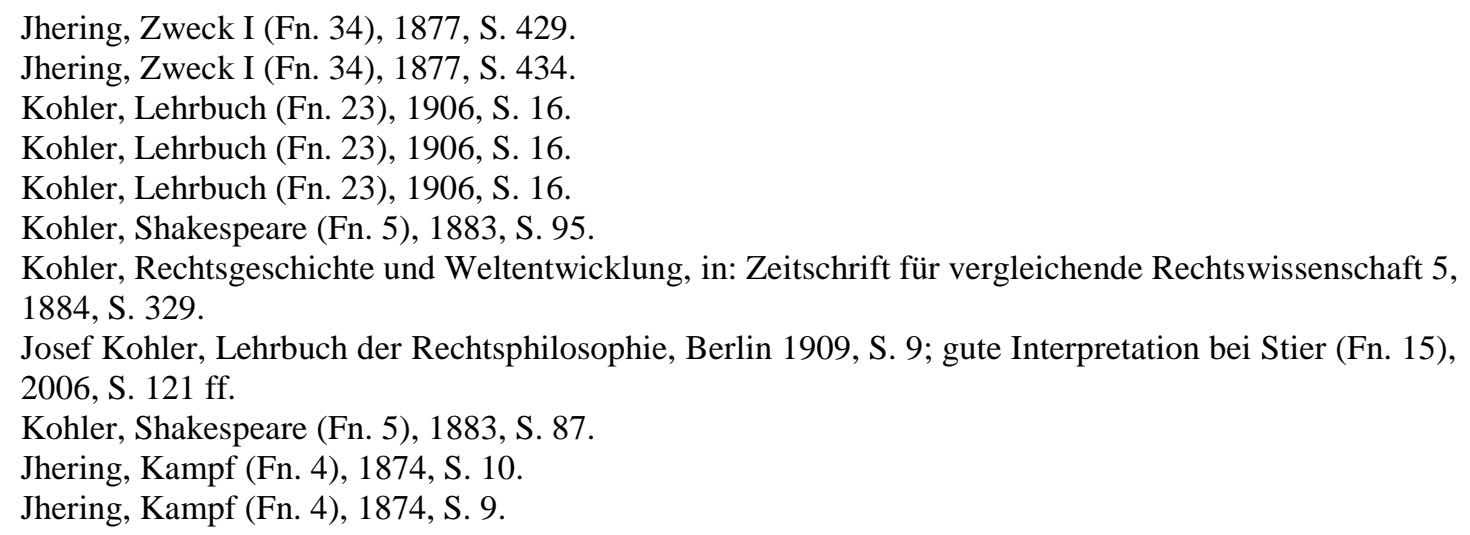




\section{JHERING, KOHLER UND DAS ERBE DER "PANDEKTENLEHRBÜCHER"}

der Bürger. Jhering glaubte durchaus an die Wahrheit einer "göttlichen Weltleitung"52. Das bedeutete jedoch keineswegs, dass der Richter historischen Wandel zulasten der Bürger herausspüren durfte. Jherings Rechtsdenken drehte sich auch in den 1870er Jahren noch um den Schutz eines gesellschaftlichen Freiheitsraumes gegen den Staat und hierfür wollte er einen anderen Richter als Kohler. Noch immer erschien ihm der Staat als eine Gefahr der individuellen Freiheit. Jherings Erfahrungen mit dem Schicksal der Göttinger Sieben ${ }^{53}$ hatten ihn gelehrt, nicht auf Verfassungen zu vertrauen, denn, so noch 1877, "man mag sie aussinnen, so künstlich wie man will, es läßt sich keine denken, welche die Staatsgewalt faktisch der Möglichkeit beraubte, das Gesetz mit Füßen zu treten"54. Gegenüber dem Gesetzgeber half nur der Appell, sich willkürlicher Eingriffe zu enthalten ${ }^{55}$. Eigentlichen Schutz gegen staatliche Willkür bot dagegen der "Kampf ums Recht". Indem der Einzelne sein gesetzlich zugesichertes subjektives Recht verteidigte, den Rechtsbruch nicht hinnahm, verteidigte er zugleich die Rechte aller, weil er Gesetzlichkeit einforderte und erzwang. Der Kampf des Individuums für das subjektive Recht war damit "Pflicht gegen das Gemeinwesen"56.

Jhering setzte also gerade im Privatrecht auf Gesetze als Sicherungen der bürgerlichen Freiheit. Das richterliche Rechtsgefühl gegen das Gesetz auszuspielen, schied für Jhering aus. Umgekehrt versuchte er Rechtsgefühl und Gesetz zu vereinen. Kurz nach Erscheinen von Kohlers Shakespeare-Buch zog Jhering in seinem Wiener Vortrag über die Entstehung des Rechtsgefühls der richterlichen Berücksichtigung des Rechtsgefühls ihren Stachel ${ }^{57}$. Das Rechtsgefühl wurde von Jhering hier nicht als Ausdruck des wie auch immer gefassten Absoluten, sondern primär als historisches Produkt der geltenden Rechtslage gedeutet. Ganz im Unterschied zu Kohler machte Jhering damit gerade das Gesetz zum entscheidenden Prägefaktor des Rechtsgefühls ${ }^{58}$. Nicht der bloße Egoismus des Einzelnen, sondern das Rechtsgefühl forderte die Einhaltung der Gesetze. Im "Kampf ums Recht" war die Verteidigung des subjektiven Rechts auch ein Aufbegehren des Rechtsgefühls, das eben die Beibehaltung des Zugesagten verlangte. War für Kohler das Rechtsgefühl Antagonist des Gesetzes, so war es für Jhering Ausdruck des Gesetzes zum Zwecke des Schutzes subjektiver Rechte.

52 Rudolph von Jhering, Geist des römischen Rechts auf den verschiedenen Stufen der Entwicklung, Bd. 1, 4., Aufl. 1878, S. 62; hierzu Rückert, Der Geist des Rechts in Jherings "Geist" und Jherings "Zweck", in: $\operatorname{Rg}$ 5, 2004, S. 141.

53 Hierzu Jherings bekannter Brief an Bismarck vom 15. 9. 1888, bei Helene Ehrenberg, Rudolf von Jhering in Briefen an seine Freunde, Leipzig 1913, S. 442.

54 Jhering, Zweck I (Fn. 34), 1877, S. 381.

55 Jhering, Zweck I (Fn. 34), 1877, S. 378: "Nur wo die Staatsgewalt selber die von ihr vorgeschriebene Ordnung befolgt, gewinnt letztere ihre Sicherheit, nur wo das Recht herrscht, gedeiht der nationale Wohlstand, blühen Handel und Gewerbe, nur da entfaltet sich die dem Volke innewohnende, geistige und moralische Kraft zu ihrer vollen Stärke".

56 Jhering, Kampf, (Fn. 4), 1874, S. 46 ff.

57 Rudolf v. Jhering, Über die Entstehung des Rechtsgefühls, mit einer Vorbemerkung und einem anschliessenden Interpretations- und Einordnungsversuch von Okko Behrends, Jovene 1986.

58 Jhering, Über die Entstehung des Rechtsgefühls (Fn. 57), 1884, S. 21 f. 


\title{
H.-P.HAFERKAMP
}

\begin{abstract}
"Das ist der Schlußpunkt der Entwicklung: das objektive, tatsächlich verwirklichte Recht und das subjektive Rechtsgefühl, beide auf derselben Höhe, beide sich gegenseitig bedingend und stützend. Auf der moralischen Macht des nationalen Rechtsgefühls beruht in letzter Instanz die ganze Sicherheit des Rechts" ${ }^{59}$.
\end{abstract}

Der streng gebundene Richter begrenzte die Eingriffe in das Privatrecht auf den Gesetzgeber. Auf den Richter als Partner der Gesellschaft gegen den Staat wollte Jhering weiterhin nicht setzen. Sein Hinweis, in ihm stecke noch zuviel von den alten Pandektenlehrbüchern, ist damit freilich weiterhin kaum erklärt. Die Pandektenlehrbücher behandelten das "Heutige Römische Recht", dessen Geltung ganz überwiegend auf Gewohnheitsrecht gestützt wurde. Gesetzesrecht war nur am Rande Thema in diesen Werken. Vor allem aber teilte man Jherings Beharren auf einer äußerst strengen Gesetzesbindung des Richters hier kaum. Dies gilt insbesondere für Puchta, dem Jhering seit Berliner Tagen viele Prägungen verdankte. Puchta hatte ihm nicht zuletzt zu seinem Buch über den "Geist des Römischen Rechts" geraten ${ }^{60}$, was Jhering $1852^{61}$ mit einer Widmung an den ja bereits verstorbenen "großen Meister" Puchta dankte. Gerade Puchta räumte dem richterlichen Rechtsgefühl jedoch viel Freiraum ein. Puchta hatte den Richter zum Partner gegen den Staat gewählt ${ }^{62}$. In der Rechtsquellenlehre und in der Auslegungslehre hatte er stets Raum gelassen für richterliche Billigkeitserwägungen und demgegenüber klassische Bindungsstrategien wie den fortwährenden Gebrauch, Präjudizien, die herrschende Meinung aber eben auch die systematische Richtigkeit abgewertet ${ }^{63}$. Bei Puchta blieb also der Volksgeist Entstehungsgrund des Rechts, das Rechtsgefühl blieb intuitiver Gerechtigkeitsappell gegenüber dem Gesetz. Zeitgenossen hatten diesen richterrechtsfreundlichen Zug in Puchtas Rechtslehre klar gesehen ${ }^{64}$ und auch Jhering dürfte er bekannt gewesen sein.

Dass Jhering sich auf die "alten Pandektenlehrbücher" berief, hatte also wohl noch einen anderen Grund. Puchta hatte bei allem Freiraum für das richterliche Rechtsgefühl stets gefordert, auch solche Entscheidungen des Billigkeitsgefühls in den Systemzusammenhang - und sei es als Ausnahme - zu integrieren und damit zu rationalisieren. In möglichst kohärenter Dogmatik lag für Puchta - neben der Person des wissenschaftlich auszubildenden Richters - eine zweite, entscheidende Stabilisierung der Autonomie des Privatrechts "gegen

\footnotetext{
59 Jhering, Zweck I (Fn. 34), 1877, S. 381.

60 Empfehlungsbrief Puchtas für Jhering an Breitkopf \& Härtel, UB der Humboldt-Universität zu Berlin, Nr. 864.

61 Rudolph von Jhering, Geist des römischen Rechts auf den verschiedenen Stufen seiner Entwicklung, Theil 1, Leipzig 1852, Widmung.

62 Dies kann hier nur angedeutet werden, vgl. nun Haferkamp, Historische Rechtsschule (Fn. 15), 2018, S. $284 \mathrm{ff}$.

63 Haferkamp, Historische Rechtsschule (Fn. 15), 2018, S. 215 ff.

64 Vgl. die Nachweise bei Haferkamp, Puchta (Fn. 22), 2004, S. 46 f.
} 


\section{JHERING, KOHLER UND DAS ERBE DER "PANDEKTENLEHRBÜCHER"}

die Staatskünstler"65. Wenn Jhering in berühmten Worten 1858 die "Form" als "die geschworene Feindin der Willkühr, die Zwillingsschwester der Freiheit" bezeichnet hatte ${ }^{66}$ dann lag er sicher mit Puchta hier auf einer Linie. Und wenn er Kohlers Hofierung nicht rational verarbeiteter Intuition seine Prägung durch Pandektenlehrbücher entgegenhielt, dann konnte sich dafür sicher besonders auf das Lehrbuch von Puchta berufen. In diesem Lehrbuch, nach dem Jhering bis $1875^{67}$ lehrte, hatte Puchta versucht, das Heutige Römische Recht als einen Zusammenhang von Prinzipien und Begriffen, mithin in extremer logischer Verdichtung zu präsentieren, was den Pandektenvorlesungen als Ort systematischen Denkens und dem Konzept des Kompendiums geschuldet war ${ }^{68}$. Dieser Darstellung hatte Jhering wohl sein bereits 1844 vorgeführtes Methodenprogramm entnommen, dass um "Prinzip" und "Consequenz" kreiste. Jhering hatte damit freilich eine Methode verabsolutiert, die Puchta ganz bewusst jenseits seines Lehrbuches nicht verfolgte ${ }^{69}$. Als Dogmatiker hatte Puchta, ganz anders als Jhering, die dogmatischen Lösungen stets auch sehr genau von der Antike bis in die Gegenwart historisch hergeleitet, präzise in der Rechtsquellenlehre verortet und zudem Aspekte wie "praktische Bedürfnisse", "natürliches Gefühl der Billigkeit" und Praxistauglichkeit neben die Arbeit mit Prinzipien gestellt ${ }^{70}$. Jhering hatte diese Auswirkungen des Volksgeistkonzepts dagegen schon früh abgeschüttelt und insbesondere die für Volksgeistdenker unaufhebbare historische Herleitung eines Satzes für regelmäßig verzichtbar erachtet ${ }^{71}$. Damit war Privatrecht im wesentlichen Dogmatik und enger in Systemzwänge eingebunden, als dies bei vorherigen und auch zeitgenössischen Pandektenwissenschaftlern der Fall war ${ }^{72}$. Schon bevor Jhering 1858 seine naturhistorische Methode formulierte, um "der Herrschaft des Rechtsgefühls ein Ende zu machen"73, hatte er in seinen frühen Abhandlungen die richtige wissenschaftliche Konstruktion höher eingestuft, als die

65 Brief Puchtas an Ludwig von Gerlach bei Hans Liermann u. Hans-Joachim Schoeps, Materialien zur preußischen Ehescheidungsreform im Vormärz, Göttingen 1961, S. 501; zum Ganzen Haferkamp, Puchta (Fn. 22), 2004, S. 434 ff.

66 Rudolph von Jhering, Geist des römischen Rechts, 2. Teil, 2. Abteilung, Leipzig 1858, S. 497.

67 Rudolf v. Jhering, Pandektenvorlesung nach Puchta, hg. Von Christian Jäde, Göttingen 2008, S. 29.

68 Genauer zu diesem Konzept: Hans-Peter Haferkamp, Methode und Rechtslehre bei Georg Friedrich Puchta (1798-1846), in: Joachim Rückert u. Ralf Seinecke, Methodik des Zivilrechts - von Savigny bis Teubner, 2. Aufl. Baden-Baden 2016, S. 73 ff.; allgemein ders., Pandektisten am Katheder, in: Claes Peterson (Hg.), Rechtswissenschaft als juristische Doktrin, Stockholm 2011, S. 85 ff.

69 Zur begrenzten Aufgabe dieser nur abgeleiteten und damit subsidiären Rechtsquelle Haferkamp, Puchta (Fn. 22), 2004, S. 429 ff.

70 Vgl. meine Analysen in Puchta (Fn. 22), 2004, S. 206 ff.

71 Vgl. bereits Jhering (anonym (Nachweis der Autorenschaft bereits bei Michael Kunze, Jherings Universalrechtsgeschichte, in: Heinz Mohnhaupt (Hg.), Rechtsgeschichte in den beiden deutschen Staaten (1988-1990), Frankfurt a. M. 1991, S. 152), Die historische Schule der Juristen, in: (Berliner) Literarische Zeitung 1844, Sp. 409: "Für jenen Zweck wird ferner ein geringes geschichtliches Material genügen...", hierzu Christoph Eric Mecke, Rudolf von Jhering. Anonym publizierte Frühschriften und unveröffentlichte Handschriften aus seinem Nachlaß, Göttingen 2010, S. 114 ff.

72 Vgl. nur die scharfe Kritik von Aloys Brinz, in: $\mathrm{KrVj}$ 2, 1860, S. 1 ff., 36: "die juristische Construction halten wir für einen Abweg; die juristische Analyse für zu eng, die Praktikabilität für zu weit angelegt."

73 Rudolph von Jhering, Geist des römischen Rechts, Bd. II 2, Leipzig 1858, S. 332. 


\section{H.-P.HAFERKAMP}

intuitive Forderung der Gerechtigkeit ${ }^{74}$. Puchta ging nie so weit und hätte Jhering wahrscheinlich unter die "neue dogmatische Schule" gerechnet, die "weder von Philosophie noch Geschichte etwas versteht, noch wissen will"75.

An dieser Stilisierung gerade der Pandektenlehrbücher als Ausdruck seiner Methode, hielt Jhering gegenüber Kohler also fest. Dass darin gefundene Programm von "Princip" und "Consequenz" blieb auch nach 1871 durchaus ein methodisches Ideal Jherings. 1884, im gleichen Jahr, in dem er seine Angriffe gegen die Begriffsjurisprudenz veröffentlichte ${ }^{76}$, ging er sogar so weit, sein Konzept von juristischer Dogmatik im Rechtsgefühl selbst zu verankern. Nachdem er das Rechtsgefühl als historisch geprägt herausgearbeitet hatte, stellte er die Frage, warum ein Rechtsgefühl, "wenn es seine Nahrung von aussen, von den Rechtssätzen und Einrichtungen, die aufgestellt sind, bezieht, schliesslich denselben überlegen wird"? Seine Antwort verwies auf das "Abstractionsvermögen des menschlichen Geistes"77. Das Rechtsgefühl führte Anwendungsfälle auf Regeln zurück und hielt diese dem Gesetzgeber als Wertungswidersprüche entgegen: "Du ziehst nicht die Consequenz Deiner Grundsätze; Du hast Grundsätze aufgestellt, aber in zu enger Fassung, die letzten Consequenzen hast Du nicht gezogen, die musst Du ziehen"78. Ganz in diesem Sinne war es ihm bereits vierzig Jahre früher darum gegangen, aus den römischen Texten Prinzipien abzuleiten, um dann "Consequenzen zu ziehen, die sie nicht gezogen"79 hatten. Im Rechtsgefühl begegneten sich also wohl nicht zufällig die beiden Schutzmechanismen, die Jhering dem Privatrecht anheim gegeben hatte: das Gesetz als prägender und das Abstraktionsvernögen als verarbeitender Teil. Dazu passt, dass Jhering bei aller Kritik am "Willensformalismus"80 das Privatrecht auch nach 1871 vom Subjekt und nicht einfach vom Staat her konstruieren wollte, wie sein Disput mit Windscheid um die Denkbarkeit subjektloser Rechte bis in die 1880er Jahre zeigte ${ }^{81}$. Auch wenn nicht zu verkennen ist, dass Jhering nach 1871 dem Reichsgesetzgeber deutlicher mehr Gestaltungsraum geben wollte, als im Deutschen Bund ${ }^{82}$, so war sein Diktum gegenüber Bismarck aus dem Jahr 1888, gegenüber der

74 Vgl. nur seine Befürchtung von 1844 "meinem Princip zu Liebe Consequenzen zugegeben zu haben, zu denen sich die römischen Juristen in ihrem Billigkeitsgefühl nicht verstanden haben würden", Jhering, Abhandlungen aus dem römischen Recht, Leipzig 1844, S. 86. Brief Puchtas an Jhering vom 8. 3. 1837, bei Horst Heinrich Jakobs, Georg Friedrich Puchta. Briefe an Gustav Hugo, Frankfurt a. M. 2009, S. 172.

76 Rudolf v. Jhering, Scherz und Ernst in der Jurisprudenz, Leipzig 1884, insb. S.330 ff.

77 Jhering, Über die Entstehung des Rechtsgefühls (Fn. 57), 1884, S. 45.

78 Jhering, Über die Entstehung des Rechtsgefühls (Fn. 57), 1884, S. 49.

79 Jhering, Abhandlungen (Fn. 74), 1844, S. VI; hierzu Ulrich Falk, Ein Gelehrter wie Windscheid, 2. Aufl. Frankfurt a. M. 1996, S. 52 ff. Beim Kohlefall änderte er dieses Verfahren nicht, fand nur ein anderes Prinzip, welches sein Gerechtigkeitsempfinden besser befriedigte, vgl. die Fallanalyse bei Okko Behrends, Das "Rechtsgefühl" in der historisch-kritischen Rechtstheorie des späten Jhering, in: Jhering, Über die Entstehung des Rechtsgefühls, 1986, S. 72 ff. und auch S. 89 f. mit ähnlichem Ergebnis wie hier.

80 Rudolph von Jhering, Geist des römischen Rechts auf den verschiedenen Stufen seiner Entwicklung, Dritter Teil. Erste Abteilung, Leipzig 1865, S. 315.

81 Hierzu Ulrich Falk, "Ein Gegensatz principieller Art", in: RJ 9, 1990, S. 220 ff.

82 Vgl. Falk, Von Dienern des Staates (Fn. 32), 1996, S. 273 ff. 
"öden Verherrlichung von Prinzipien und tote Formeln hoffe ich auf den Segen einer gewaltigen Persönlichkeit", doch auch eine jheringstypische Zuspitzung. Für Jhering blieb es die Aufgabe juristischer Dogmatik, den juristisch-rationalen Fachdiskurs von vagen Billigkeitsformeln abzugrenzen und so die Autonomie des Privatrechts zu schützen. Jedenfalls insofern steckte "noch zu viel von der alten Jurisprudenz aus den ,Pandektenlehrbüchern'" in ihm, um Kohlers Träume vom Richter als Medium des kulturellen Fortschritts im Privatrecht mittragen zu können.

Hans-Peter Haferkamp ${ }^{83}$

Köln

83 H.-P. Haferkamp ist Professor Bürgerliches Recht, Neuere Privatrechtsgeschichte und Deutsche Rechtsgeschichte an der Universität zu Köln. 
H.-P.HAFERKAMP

GROM (2018) 